\title{
Comparação de métodos de avaliação nutricional empregados no acompanhamento de pacientes submetidos a transplante de células-tronco hematopoéticas alogênico
}

\author{
Comparison of nutritional assessment methods employed to monitor patients undergoing \\ allogeneic stem cell transplantation
}

Heloisa M. Sommacal

Ana Maria K. Jochims²

Ilaine Schuch ${ }^{3}$

Lúcia M. R. Silla
Pacientes submetidos a transplante de células-tronco hematopoéticas alogênico (TCTH alo) exigem atenção diferenciada devido à agressividade do tratamento. O objetivo desse trabalho foi comparar os métodos disponiveis e a frequência de avaliações nutricionais nesses pacientes. Dezessete pacientes foram avaliados semanalmente entre o dia $D-7$ e D+28, divididos em cinco intervalos. Foram avaliados altura, peso, indice de massa corporal (IMC), circunferência do braço (CB), dobra cutânea tricipital $(D C T)$, circunferência muscular do braço $(C M B)$, percentual de perda de peso $(\% P P)$, dosagem de albumina sérica, balanço hídrico total (BHT), controle de ingestão e percentual de massa magra (MM), massa gorda $(M G)$ e água $\left(\mathrm{H}_{2} \mathrm{O}\right)$ através de bioimpedância elétrica. A média de consumo energético foi de 1.377 (DP $\pm 582 \mathrm{Kcal}$ ). $76,47 \%(n=13)$ deles tiveram diminuição de peso e, desses, $57 \%(n=8)$ foram classificados como significativo; a média de \%PP foi de 6,27 Kg (DP $\pm 4,25)$. A CB mostrou diferença significativa $(p<0,05)$ entre cinco intervalos, mostrando-se a mais sensivel das medidas antropométricas. Os \% MM e \% MG mostraram diferença significativa $(p<0,05)$ entre quatro intervalos. Pela correlação de Pearson mostrou-se significativa $(p<0,01)$ para as variáveis: peso X CB; CB X DCT; $C B X C M B ; C B X I M C ; M M X$ $M G$; $M M \mathrm{X} \mathrm{H}_{2} \mathrm{O}$; $\mathrm{BHT} \mathrm{X} \mathrm{H}_{2} \mathrm{O}$. O percentual de perda de peso se mostrou o mais sensivel dos métodos avaliados, e, associado às avaliações de DCT e CB quinzenais, é adequado para o acompanhamento da evolução do estado nutricional. Rev. Bras. Hematol. Hemoter. 2010;32(1):xx-xx.

Palavras-chave: Antropometria; composição corporal; peso e medidas corporais; transplante de medula óssea; transplante de células-tronco hematopoéticas.

\section{Introdução}

Devido à intensa agressividade da terapia do condicionamento no transplante de células-tronco hematopoéticas alogênico (TCTH alo), os pacientes sofrem lesões nas barrei- ras cutâneas e mucosas com graves consequências e elevado risco de desnutrição. No paciente oncológico em geral, e no transplantado em particular, a desnutrição aumenta a incidência de infecções além de diminuir a tolerância à quimioterapia. ${ }^{1,2} \mathrm{~A}$ intervenção nutricional pode diminuir a

\footnotetext{
${ }^{I}$ Nutricionista. Pesquisadora, Hospital de Clínicas de Porto Alegre-RS

${ }_{3}^{2}$ Nutricionista do Hospital de Clínicas de Porto Alegre-RS.

${ }^{3}$ Nutricionista. Professora Assistente da Universidade Federal do Rio Grande do Sul - Porto Alegre-RS.

${ }^{4}$ Professora Adjunta do Departamento de Medicina Interna da Universidade Federal do Rio Grande do Sul. Chefe do Serviço de

Hematologia e Transplante de Medula Óssea do Hospital de Clínicas de Porto Alegre-RS.
}

Hospital de Clínicas de Porto Alegre-RS.

Correspondência: Heloisa Sommacal

Rua Jacinto Gomes, 260/102 - Santana

90040-270 - Porto Alegre/RS - Brasil

Tel.: (51) 3311-5110

E-mail:helo.s@uol.com.br 
toxicidade e melhorar a sobrevida nestes pacientes, ${ }^{3}$ e as Unidades de TCTH desenvolvem protocolos assistenciais com o objetivo de evitar complicações graves ou irreversíveis durante a internação. O Hospital de Clínicas de Porto Alegre (HCPA), seguindo as tendências, desenvolveu, em 1999, seu protocolo baseado no controle de ingestão, avaliações antropométricas e bioquímicas, semanais, no sentido de propiciar uma intervenção precoce. A antropometria e a bioimpedância têm o objetivo de identificar as mudanças físicas que ocorrem no corpo, dentre elas a proporção de massa magra e massa gorda, quantidade de água, perda ou ganho de peso. O método de bioimpedância tem sido relatado como simples, não invasivo, relativamente barato e de fácil aplicação por ser portátil. ${ }^{4,5}$ As avaliações individuais e periódicas, o controle de ingestão, o balanço hídrico total e exames bioquímicos permitem acompanhar e detectar rapidamente uma possível desnutrição assim como a presença de edema por acúmulo de líquidos. Devido à limitação de cada método para avaliação nutricional (não há estabelecido um padrão ouro), existe a necessidade da utilização de dois ou mais instrumentos de avaliação nutricional a fim de estabelecer a combinação mais eficiente e menos onerosa ao paciente. Porém, apesar de essencial, a grande manipulação desses pacientes, representada pela utilização de vários métodos de aferição nutricional, tem sido relatada em trabalhos recentes como um fator negativo do ponto de vista do paciente. ${ }^{6}$ Finalmente, a hipoalbunemia pode estar relacionada com a desnutrição e, portanto, a dosagem desta proteína parece importante. Embora a sensibilidade deste parâmetro não seja considerada ideal em função de sua longa meia-vida (18 a 20 dias), é um índice bastante utilizado na prática médica devido ao baixo custo. Além disso, vem se mostrando eficiente como indicativo de prognóstico nutricional e risco para complicações durante a internação. ${ }^{7}$

Assim, o objetivo deste estudo foi verificar em nosso meio qual método (antropométrico, bioimpedância ou bioquímico) e frequência de aferição ideais são necessários e suficientes para garantir um estado nutritivo adequado em pacientes submetidos ao transplante de células-tronco hematopoéticas.

\section{Casuística e Método}

Estudo observacional, incluindo 17 pacientes maiores de 18 anos submetidos ao TCTH-alo no HCPA, na Unidade de Ambiente Protegido, no período de maio de 2006 a dezembro de 2007. Todos os transplantados, neste período, com idade igual ou superior a 18 anos foram incluídos. O período analisado foi do dia $\mathrm{D}-7$ ao dia $\mathrm{D}+28$, quando a maioria dos pacientes permanece internada, sendo que o dia D 0 é o dia da infusão de células-tronco hematopoéticas. Esse período foi dividido em cinco intervalos de sete dias. Métodos de aferição: antropometria (1) massa corporal (balança antropométrica da marca Filizola ${ }^{\circledR}$ com capacidade para $150 \mathrm{~kg}$ e variação de 0,050 gramas); (2) Altura (estadiômetro vertical marca Sanny ${ }^{\circledR}$, com variação em centímetros); (3) Índice de Massa Corporal - IMC (Organização Mundial da Saúde, 1998) $;{ }^{8}$ Percentual de perda de peso $(\% \mathrm{PP}=$ peso usual peso atual x 100/peso usual, e classificado segundo Blackburn ${ }^{9}$ e (4) Circunferência do braço, circunferência muscular do braço e dobra cutânea tricipital. Estas últimas medidas foram realizadas da seguinte maneira: o paciente de pé, com o braço relaxado na lateral do corpo e palma da mão voltada para coxa. A marca do ponto médio realizou-se com fita graduada medindo o ponto médio entre o acrômio e o olecrano, com o braço flexionado junto ao corpo. ${ }^{7,8} \mathrm{~A}$ fita foi colocada em torno do ponto médio do braço e o valor da circunferência confrontado com os valores referentes a sexo e idade,$^{10}$ e adequados pelas porcentagens propostas por Blackburn. ${ }^{9}$ Foram utilizados plicômetro medidor de dobras cutâneas científico e fita antropométrica inelástica, com escala em milímetros, com comprimento total de $150 \mathrm{~cm}$, da marca Cescorf ${ }^{\circledR}$. A dobra cutânea tricipital foi mensurada na parte posterior do braço, com os braços relaxados e estendidos ao longo do corpo, à meia distância entre o acrômio e o olécrano, no sentido das fibras musculares. A medida da DCT era repetida três vezes e realizada a média delas. Quando as medidas diferiam $\mathrm{em} \pm 5 \%$ era realizada uma nova medição. ${ }^{7,8}$ Para a medida da circunferência muscular do braço foram utilizados os resultados obtidos pela $\mathrm{CB}$ e DCT. ${ }^{7,8}$ As medidas de bioimpedância foram executadas no lado direito do corpo. O aparelho é conectado aos dois pares de eletrodos, onde uma corrente de baixa voltagem é passada pelo corpo, sendo medidas a resistência e a reactância elétrica. Os valores obtidos, combinados com altura, peso e sexo, possibilitam o cálculo de compartimentos corporais. ${ }^{4,5,8} \mathrm{O}$ aparelho utilizado era da marca Bodystat $1500 \AA$. Todas estas aferições foram realizadas por profissional da enfermagem treinado para este fim. O controle de ingestão de alimentos foi realizado pela pesagem (balança digital Urano ${ }^{\circledR}$ ) dos alimentos oferecidos antes e após as refeições e pelo cálculo dos micro e macronutrientes ingeridos através do Programa de Apoio à Nutrição - Nutrii ${ }^{\circledR}$, versão 2.5 . (Programa de computador Sistema de Apoio à decisão em Nutrição, versão 2.5 Centro de Informática em Saúde de Universidade Federal de São Paulo de Medicina (CIS-Unifesp-EPM). Para a medida da albumina sérica foram utilizados todos os valores dosados, média de três dosagens por paciente, durante a internação. Os valores encontrados nos prontuários foram comparados com valores de referência. ${ }^{2}$ A técnica utilizada para a medida da albumina sérica foi colorimetria verde de bromocresol.

\section{Análise estatística}

Foi realizada uma análise descritiva utilizando cálculo de médias, desvio padrão, frequência e percentual através dos pacotes estáticos Statistical Package for the Social Science (SPSS), expressos através de gráficos e tabelas. O teste de Pearson foi realizado para analisar as variáveis con- 
tínuas. Foram considerados estatisticamente significativos os valores de $p$ menores que 0,05 . Foi utilizado também o teste $\mathrm{t}$ para amostras pareadas, onde foram considerados estatisticamente significativos valores de $p$ menores que 0,05 . O estudo foi aprovado pela comissão científica e a comissão de pesquisa e ética em saúde do HCPA.

\section{Resultados}

Dos 17 pacientes incluídos, seis eram do sexo feminino e 11 do sexo masculino. De acordo com o tipo de transplante, 11 foram TCTH-alo relacionados e seis não relacionados. A média do tempo de internação foi de 39 dias (DP \pm 12 ). Rzepecki et al. encontraram média de 27 dias (min 17 e max 58), ${ }^{11}$ em um estudo envolvendo transplantados alogênicos e autólogos; Bechard et al. encontraram média de 38 dias entre pacientes alogênicos. ${ }^{12} \mathrm{~A}$ Tabela 1 mostra a estatística descritiva de algumas variáveis da amostra.

Das doenças tratadas, a leucemia mieloide crônica aparece com 29\% $(n=5)$ dos casos; em seguida, a leucemia mieloide aguda com $24 \%(n=4)$, leucemia linfoide aguda com $18 \%(n=3)$, aplasia e síndrome mielodisplásica com $12 \%$ $(n=2)$ e doença de Hodgkin com $6 \%(n=1)$. Todos passaram por tratamento quimioterápico e/ou radioterápico antes do TCTH.

A média de \%PP foi de 5,05 kg (DP $\pm 2,77)$ em 41,3 dias $(\mathrm{DP} \pm 5,71)$ entre as mulheres e de 7,02 kg $(\mathrm{DP} \pm 5,62)$ em 38,36 dias $(\mathrm{DP} \pm 14,88)$ entre os homens. O percentual de perda de peso durante o período analisado está demonstrado no Gráfico 1.

Dentro dos valores de índice de massa corporal (IMC), $33 \%$ das mulheres $(n=2)$ e $27 \%$ dos homens $(n=3)$ mudaram de classificação durante o tratamento, sendo que $18 \%$ dos pacientes $(n=3)$ passaram de sobrepeso a eutrofia, $6 \%(n=1)$ de obesidade grau I para sobrepeso e $6 \%(n=1)$ de obesidade grau II para obesidade grau I.

As medidas de circunferência do braço, da dobra cutânea triciptal e circunferência muscular do braço foram analisadas pelo teste $t$ para amostras pareadas (Tabela 3). A CB mostrou diferença significativa $(p<0,05)$ entre cinco intervalos, mostrando-se a mais sensível das três medidas (primeira mudança foi entre a avaliação 1 e 3). A DCT apresentou diferença significativa $(p<0,05)$ entre três intervalos (primeira mudança entre 1 e 3 ), diferente de outros estudos que utilizaram as mesmas variáveis, porém a CMB não mostrou diferença significativa, assim como nos estudos de Burkinshaw, Cheney, Rzepecki, Shizgal. ${ }^{11,13-15}$

Nossos resultados também se assemelham a outros estudos, como o de Garófolo, ${ }^{16}$ realizado em crianças, porém mostrando variações maiores entre

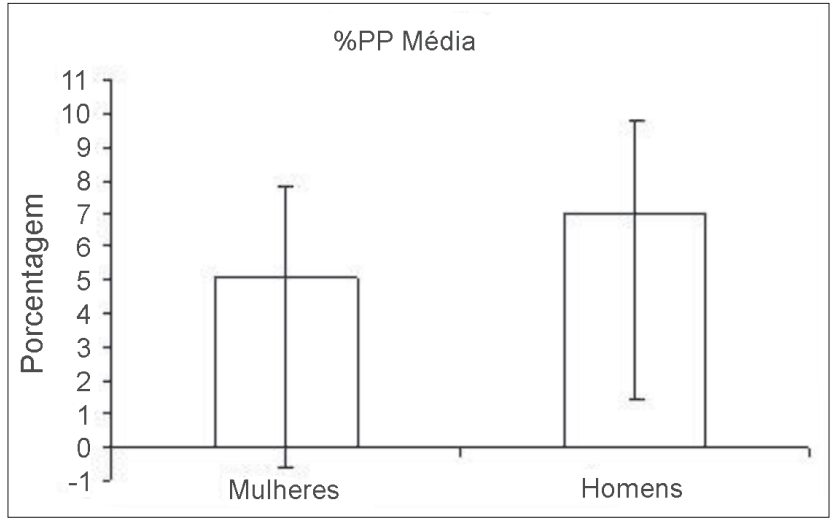

Gráfico 1. Percentual de perda de peso (\%PP) entre mulheres e homens no intervalo analisado (D-7 e D+28)
DCT, CMB e CB que em IMC. Encontramos diminuição de massa gorda e aumento de massa magra em 94\% $(n=16)$ dos pacientes, a variação média está descrita na Tabela 2).

Aplicou-se o teste t para amostras emparelhadas nas medidas repetidas de bioimpedância quanto à porcentagem de massa magra, massa gorda e porcentagem de água (Tabela 3). A \%MM e \%MG mostraram diferença significativa $(\mathrm{p}<0,05)$ entre quatro intervalos (sendo o primeiro entre a avaliação 1 e 2).

O percentual da água foi analisado apesar da superhidratação recebida por esses pacientes e encontramos variação significativa em quatro intervalos (sendo o primeiro entre a avaliação 1 e 2).

Na análise pela correlação de Pearson entre a avaliação de água pelo método de bioimpedância e pelo controle do 
Tabela 3. Resultados da anlise do teste t para amostras pareadas,entre os intervalos de tempo analisados $(D-7$ ao $D+28)$

\begin{tabular}{|c|c|c|c|c|c|c|c|c|c|c|c|}
\hline \multirow[b]{2}{*}{$\begin{array}{c}\text { Avaliação entre } \\
\text { os intervalos }\end{array}$} & \multicolumn{6}{|c|}{ Antropometria } & \multicolumn{5}{|c|}{ Bioimpedância } \\
\hline & IMC & $\%$ PP & $\mathrm{CB}$ & $\mathrm{CMB}$ & DCT & $\% \mathrm{MM}$ & $\% \mathrm{MG}$ & $\% \mathrm{H} 2 \mathrm{O}$ & Kcal & Alb & Peso \\
\hline 1 e 2 & NS & NS & NS & NS & NS & $p<0,000$ & $p<0,001$ & $p<0,000$ & $p<0,001$ & $p<0,005$ & NS \\
\hline 1 e 3 & NS & NS & $p<0,000$ & NS & $p<0,012$ & $p<0,007$ & $p<0,006$ & $p<0,005$ & $p<0,000$ & $p<0,005$ & NS \\
\hline 1 e 4 & $p<0,009$ & $N S$ & $p<0,002$ & NS & $p<0,004$ & $p<0,001$ & $p<0,001$ & $p<0,001$ & $p<0,001$ & $p<0,000$ & $p<0,009$ \\
\hline 1 e 5 & $p<0,045$ & $p<0,049$ & $p<0,028$ & NS & $p<0,013$ & $p<0,001$ & $p<0,001$ & $p<0,001$ & $p<0,010$ & NS & $p<0,008$ \\
\hline 2 e 3 & $p<0,029$ & $p<0,015$ & $p<0,014$ & NS & NS & NS & NS & NS & $p<0,019$ & NS & $p<0,029$ \\
\hline 2 e 4 & $p<0,001$ & $p<0,024$ & $p<0,006$ & NS & NS & NS & NS & NS & NS & NS & $p<0,001$ \\
\hline 2 e 5 & $p<0,023$ & $p<0,004$ & NS & NS & NS & NS & NS & NS & NS & NS & $p<0,003$ \\
\hline 3 e 4 & $p<0,011$ & NS & NS & NS & NS & NS & NS & NS & NS & NS & $p<0,020$ \\
\hline 3 e 5 & NS & $p<0,029$ & NS & NS & NS & NS & NS & NS & $p<0,042$ & NS & $p<0,016$ \\
\hline 4 e 5 & NS & $p<0,015$ & NS & NS & NS & NS & NS & NS & NS & NS & NS \\
\hline
\end{tabular}

NS: Diferença não significativa; IMC: índice de massa corporal; \%PP: percentual de perda de peso; CB: circunferência do braço;

CMB: circunferêncmuscular do braço; DCT: dobra cutânea tricipital; \%MM: percentual de massa magra; \%MG: percentual de massa gorda;

$\% \mathrm{H}_{2} \mathrm{O}$ : Percentual de água; Kcal: calorias; Alb: Albumina

balanço hídrico, foram significativas $(\mathrm{p}<0,05)$ as diferenças no $2^{\circ}$ e $3^{\circ}$ intervalos.

Pela correlação de Pearson mostrou-se significativa $(\mathrm{p}<0,01)$ para as variáveis: peso $\mathrm{X} \mathrm{CB}$; CB X DCT; CB X CMB; CB X IMC; MM X MG; MM X $\mathrm{H}_{2} \mathrm{O}$ e BHT X $\mathrm{H}_{2} \mathrm{O}$.

Os primeiros indicadores a mostrar alterações significativas $(\mathrm{p}<0,05)$ foram dosagem de albumina sérica, porcentagem de massa magra e porcentagem de massa gorda, quando medidas pelo método de bioimpedância e o controle de ingestão, entre o $1^{\circ}$ e o $2^{\circ}$ intervalos. Porém, esses métodos não mantiveram alterações significativas entre os intervalos restantes. Entre o $2^{\circ}$ e o $3^{\circ}$ intervalos, cinco indicadores mostraram alterações significativas: IMC, CB, peso, \%PP e controle de ingestão. Entre o $3^{\circ}$ e $4^{\circ}$ intervalos, o peso e o IMC mostraram alterações significativas. No $4^{\circ}$ intervalo para o $5^{\circ}$, apenas o \% PP (Tabela 3).

Quando analisados em relação à primeira e à última avaliação, encontramos mudança significativa nas variáveis IMC, \%P, CB, DCT, \%MM, \%MG, \% $\mathrm{H}_{2} \mathrm{O}$, ingestão em Kcal e variação de peso (Tabela 3 ).

Pelo controle de ingestão analisamos o consumo médio de ingestão dos pacientes e a porcentagem de macronutrientes (Tabela 4).

A dosagem de albumina sérica é utilizada como um parâmetro inicial na avaliação nutricional desses pacientes; ${ }^{18}$ também foi acompanhada e analisada pelo teste t e mostrou diferença significativa $(p<0,05)$ em três intervalos (primeira mudança entre avaliação 1 e 2) (Tabela 3). Apesar de ter vida útil em torno de 21 dias, a dosagem de albumina apresentou variação nesses pacientes abaixo desse período, necessitando de maiores estudos.

Dos 17 pacientes, 58,90\% $(\mathrm{n}=10)$ usaram nutrição parenteral total (NPT) e 41,10\% (n=7) não utilizaram nenhuma forma de suporte nutricional.

\section{Discussão}

Quanto à ingestão calórica, segundo a literatura, as recomendações para pacientes submetidos à TCTH-alo é de $130 \%$ a $150 \%$ do gasto energético basal segundo Harris, (GEB/ HB). ${ }^{17-21}$ Entre os pacientes estudados, este valor representaria entre 1.575 a $1.816 \mathrm{Kcal}$ entre as mulheres e 1.398 a 1.614 Kcal entre os homens. Porém, encontramos a média de consumo energético de $1.073 \mathrm{Kcal}$ e $1.543 \mathrm{Kcal}$ entre mulheres e homens, respectivamente, ficando em $89 \%$ do GEB/HB pelas mulheres e $113 \%$ do GEB/HB pelos homens. A quantidade de proteína recomendada está entre 1,3 e 2g/ $\mathrm{kg} \cdot{ }^{17,20,21}$ Em nosso estudo encontramos a média de $0,53 \mathrm{~g}$ de proteína $/ \mathrm{kg}, 40 \%$ do valor mínimo recomendado, entre as mulheres e $0,65 \mathrm{~g}$ de proteína/kg entre homens, $50 \%$ do valor mínimo recomendado.

Para o uso de NP, cada estudo segue o protocolo da
Tabela 4. Média de ingestão durante o período analisado (D-7 ao D+28)

\begin{tabular}{ccccccccc}
\hline & \multicolumn{2}{c}{ Kcal } & \multicolumn{2}{c}{$\%$ CHO } & \multicolumn{2}{c}{$\%$ LIP } & \multicolumn{2}{c}{$\% N P T$} \\
\hline & Média & DP \pm & Média & DP \pm & Média & DP \pm & Média & DP \pm \\
\hline Mulheres & 1073 & 472 & 54,33 & 14,68 & 20,84 & 1,69 & 12,18 & 3,06 \\
Homens & 1543 & 587 & 59,97 & 5,94 & 20,84 & 1,69 & 12,6 & 1,18 \\
\hline
\end{tabular}

$\mathrm{Kcal}=$ quilo calorias; $\% \mathrm{CHO}=$ percentual de carboidratos;

$\%$ LIP = percentual de lipídios; $\%$ NPT = percentual de proteínas unidade analisada, podendo variar o percentual de pacientes que fazem uso de NPT. Rzepecki encontrou 33\% de transplantados alogênicos que necessitaram de NPT; ${ }^{11}$ Arizmendi encontrou de $58 \%$ a $92 \%$ de uso de NPT, dependendo da forma de condicionamento realizado. ${ }^{22}$ Pela sua baixa sensibilidade, a medida de 
IMC não é indicada para avaliar nutricionalmente esse tipo de paciente, nem em pacientes com tumores sólidos, que apresentam maior risco de desnutrição que pacientes com neoplasias hematológicas. ${ }^{16}$ Apesar disso, muitos protocolos utilizam o IMC como parâmetro, tanto para o início quanto para o fim, do uso de NPT. ${ }^{20,23,25} \mathrm{O} \%$ PP também é utilizado como parâmetro para o uso de suporte nutricional. ${ }^{11,17,20}$

As medidas de circunferência do braço, da dobra cutânea triciptal e circunferência muscular do braço foram analisadas pelo teste t para amostras pareadas (Tabela 3). A CB mostrou diferença significativa $(p<0,05)$ entre cinco intervalos, mostrando-se a mais sensível das medidas antropométricas (primeira mudança foi entre a avaliação 1 e 3 ). DCT apresentou diferença significativa $(p<0,05)$ entre três intervalos (primeira mudança entre 1 e 3 ), diferente de outros estudos que utilizaram as mesmas variáveis; porém, a CMB não mostrou diferença significativa, assim como esses mesmos estudos. ${ }^{11,13-15}$ Nossos resultados se assemelham a outros estudos, como o de Garófolo, realizado em crianças, porém mostrando variações maiores entre $\mathrm{DCT}, \mathrm{CMB}$ e $\mathrm{CB}$ que em IMC. ${ }^{16}$

Apesar de pouco indicado para pacientes com hiperhidratação $0^{4,5,25}$ foi utilizada a bioimpedância em nosso estudo e encontramos diminuição de massa gorda e aumento de massa magra em $94 \%(n=16)$ dos pacientes.

\section{Conclusão}

Nenhum método de avaliação nutricional, antropométrico ou bioquímico, é livre de falhas e/ou contraindicações. Cada unidade deve pesquisar e identificar o mais adequado para sua realidade, evitando assim o excesso de manipulação desses pacientes. Nesse trabalho encontramos resultados semelhantes entre $\mathrm{CB}$, DCT e bioimpedância, podendo ser utilizados isoladamente. O IMC e a dosagem de albumina sérica não se mostram bons marcadores e o \% PP demonstra um sensível indicador aparente.

Sugerimos assim a utilização de um método de medição de massa corporal, dobras cutâneas ou bioimpedância, e com intervalos maiores, quinzenalmente, visto que a diminuição do número de avaliações não prejudica a evolução do paciente.

\footnotetext{
Abstract

Patients undergoing hematopoietic stem cell transplantation require special attention due to the aggressiveness of treatment. The aim of this study was to compare methods used to monitor nutritional status (including anthropometric methods of bioelectrical impedance analysis, dietary intake and biochemical control) and the frequency of nutritional evaluations in these patients. Weekly assessments were performed for six female and eleven male patients between day (D)-7 and D+28, divided into 5 time intervals. The height, weight, triceps skinfold (TS), arm circumference (AC), arm muscle
}

circumference $(A M C)$, percentage of weight loss (\%WL), concentration of serum albumin, body mass index (BMI), total water balance (TWB) and control of intake were evaluated. Additionally, the percentages of lean mass (LM), fat mass (FM) and water $\left(\mathrm{H}_{2} \mathrm{O}\right)$ were assessed by electric bioimpedance. The average daily food intake was $1377 \pm 582 \mathrm{Kcal}$. Of the total, $76.47 \%(n=13)$ of patients suffered weight loss with $57 \%(n=8)$ of weight loss being classified as significant. Among the different anthropometric measurements, arm circumference was the most sensitive with significant differences between the 5 time intervals. Additionally, there were significant differences for the percentages of lean mass $(p<0.05)$ and for fat mass $(p<0.05)$. Using the Pearson correlation, significant differences $(p<0.01)$ were calculated for the following variables: weight vs. $A C, A C$ vs. TS, $A C$ vs. $A M C, A C$ vs. BMI, LM vs. FM, $L M$ vs. $\mathrm{H}_{2} \mathrm{O}$ and $\mathrm{H}_{2} \mathrm{O}$ vs. TWB. According to the results, the percentage of weight loss in association with fortnightly DCT and CB assessments are the best way to monitor the nutritional status. Rev. Bras. Hematol. Hemoter.

Key words: Anthropometry; body composition;weight and body measurements; bone marrow transplantation; hematopoetic stem cell transplantation.

\section{Referências Bibliográficas}

1. Borelli P, Blatt SL, Rogero MM, Fock R. Haematological alterations in protein malnutrition. Revista Brasileira de Hemoterapia, 2004(26):49-56.

2. Waitzberg DL. Nutrição Oral, Enteral e Parenteral na Prática Clínica. $3^{\mathrm{a}}$ ed. Brasil; Atheneu; 2001.

3. MN Harvie MN, Howell A, Thatcher N, Baildam, Campbell I. Energy balance in patients with advanced NSCL, metastatic melanoma and metastatic breast cancer receiving chemotherapy a longitudinal study. Br J Cancer. 2005;92(4):673-80.

4. Kyle UG, Bosaeus I, De Lorenzo AD, Deurenberg P, Elia M, Gómez $\mathrm{JM}$, et al. ESPEN Guidelines: Bioelectrical impedance analysispart I: Review of principles and methods. Clin Nutr. 2004; 23 (5):1226-43

5. Kyle UG, Bosaeus I, De Lorenzo AD, Deurenberg P, Elia M, Gómez $\mathrm{JM}$, et al. ESPEN Guidelines: Bioelectrical impedance analysispart II: Utilization in clinical practice. Clin Nutr. 2004; 23(6): 1430-53.

6. Pontes L, Guirardello EB, Campos CJG. Demandas de atenção de um paciente na unidade de transplante de medula óssea. Rev. Esc. Enfermagem USP, 2007;41(1):154-60.

7. Duarte ACG. Avaliação Nutricional - Aspectos clínicos e laboratoriais, $1^{\text {a }}$ ed., Atheneu, 2007;41-76.

8. WHO. Physical status: the use and interpretation of anthropometry. Report of a WHO Expert Committee. WHO Technical Report Series 854. Geneva: World Health Organization, 1995.

9. Blackburn GL, Thornton PA. Nutritional assessment of the hospitalized patients. Med Clin North Am. 1979;63(5):11.103-15.

10. Frisancho AR. New norms of upper limb fat and muscle areas for assessment nutritional status. Am J Clin Nutr. 1981;34(11):2540-5.

11. Rzepecki P, Barzal J, Sarosiek T, Oborska S, Szczylik C. Which parameters of nutritional status should we choose for nutritional assessment during hematopoietic stem cell transplantation? Transplant Proc. 2007;39(9):2902-4.

12. Bechard LJ, Guinan EC, Feldman HA, Tang V, Duggan C. Prognostic factors in the resumption of oral dietary intake after allogeneic 
hematopoietic stem cell transplantation (HSCT) in children. JPEN J Parenter Enteral Nutr. 2007;31(4):295-301.

13. Burkinshaw HM, Jones DW, Krupowicz DW. Observer error in skinfolds thickness measurements. Hum Biol. 1973;45(2):273-9.

14. Cheney CL, Abson KG, Aker SN, Lenssen P, Cunningham BA, Buergel NS, et al. Body composition changes in marrow transplant recipients receiving total parenteral nutrition. Cancer. 1987;59 (8): 1515-9.

15. Shizgal HM. Validation of the measurement of body composition from whole body bioelectric impedance. Infusionstherapie. 1990;17 Suppl 3:67-74.

16. Garófolo A, Lopez FA, Petrilli AS. High prevalence of malnutrition among patients with solid non-hematological tumors as found by using skinfold and circumference measurements. Sao Paulo Med J. 2005;123(6):277-81..

17. Raynard B, Nitenberg G, Gory-Delabaere G, Bourhis JH, Bachmann $\mathrm{P}$, Bensadoun RJ, et al. Summary of the Standards, Options and Recommendations for nutritional support in patients undergoing bone marrow transplantation (2002). Br J Cancer. 2003;89 Suppl 1:S101-6.

18. Harris JA, Benedict PG. A Biometric Study of Basal Metabolism in Man. Carnegie Institution: Washington, 1919(279).

19. Iestra JA, Fibbe WE, Zwinderman AH, van Staveren WA, Kromhout D. Body weight recovery, eating difficulties and compliance with dietary advice in the first year after stem cell transplantation: a prospective study. Bone Marrow Transplant. 2002;29(5):417-24.

20. Martin-Salces M, Paz R, Canales MA, Mesejo A, HernandezNavarro F. Nutritional recommendations in hematopoietic stem cell transplantation. Nutrition. 2008;24(7-8):769-75.

21. Muscaritoli M, Grieco G, Capria S, Iori AP, Rossi Fanelli F. Nutritional and metabolic support in patients undergoing boné marrow transplantation. Am J Clin Nutr. 2002;75(2):183-90.

22. Arizmendi MA. Nutrición artificial em el transplante de células precursoras hematopoyéticas. Nutricion Hospitalaria. 2005(20) supp. 2:54-56.

23. Álvarez MEG. La nutrición parenteral en el transplante de células progenitoras hematopoyéticas. Farmacia Hospitalaria. 2005(28): 116-22.

24. Lipkin AC, Lenssen P, Dickson J. Nutrition issues in hematopoietic stem cell transplantation: State of the art. Nutr Clin Pract. 2005; 20(4):423-39.

25. Kyle UG, Piccoli A, Pichard C. Body composition measurements: interpretation finally made easy for clinical use. Curr Opin Clin Nutr Metab Care. 2003;6(4):387-93.

Avaliação: Editor e dois revisores externos

Conflito de interesse: sem conflito de interesse

Recebido: 16/06/2009

Aceito: $21 / 08 / 2009$ 